\title{
The SOC Estimation of Power Li-Ion Battery Based on ANFIS Model*
}

\author{
Tiezhou Wu, Mingyue Wang, Qing Xiao, Xieyang Wang
}

School of Electrical \& Electronic Engineering, Hubei University of Technology, Wuhan, China.

Email: wtz315@163.com, yueyueming@yahoo.cn

Received September $5^{\text {th }}, 2011$; revised October $11^{\text {th }}, 2011$; accepted October $18^{\text {th }}, 2011$

\begin{abstract}
On basis of traditional battery performance model, paper analyzed the advantage and disadvantage of SOC estimation methods, introduced Adaptive Neuro-Fuzzy Inference Systems which integrated artificial neural network and fuzzy logic have predicted SOC of battery. It's a battery residual capacity model with more generalization ability, adaptability and high precision. By analyzing the battery charge and discharge process, the key parameters of SOC are determined and the experimental model is modified in MATLAB platform.Experimental results show that the difference of SOC prediction and actual SOC is below 3\%. The model can reflect the characteristics curve of the battery. SOC estimation algorithm can meet the requirements for precision. The results have a high practical value.
\end{abstract}

Keywords: State of Charge; ANFIS; Estimation Method; Li-Ion Battery

\section{Introduction}

Battery is an energy source for electric vehicles. In order to make sure the good performance of the battery pack and extend it's life, it is necessary to make good control and management for the battery pack. Li-ion battery has the advantages of high voltage, high energy, long cycle life, low self-discharge, no memory effect, no pollution etc [1]. So it gradually replaces the others in electric vehicles and hybrid electric vehicle.

In the light of the battery voltage, current, temperature and other real-time online measurement, the online and real-time estimation about the SOC is possible. The value of SOC directly reflects the state of the battery, it is appropriate to tell the different performances between the various cells in the battery pack by the different SOC value of each cell, and the balanced charge can be operated according the different performances. The final aim is to extend the battery life, limit the maximum discharge current and predict the driving range of electric vehicles etc [2]. There is much improved room in the application of battery SOC online and real-time estimation method. It is impossible to meet the actual requirements as the error of estimation more than $8 \%$ [3]. To improve the accuracy of SOC online and real-time esti-

\footnotetext{
*This work was supported by natural science foundation of Hubei province (research on soc estimation method and equalizing charging of lithium-ion battery for HEV. No. ZRY1530). Author: Tiezhou Wu (1966-), male, associate professor, mater tutor, Research direction: signal analysis and system integration.
}

mation, intensive study is necessary in measurements method, the battery model and estimation method.

\section{Traditional SOC Estimation Method}

SOC show that the percentage of remaining capacity in marker capacity [4], and that is defined as the rate of remaining and the ratio capacity. The range is from $0 \%$ to $100 \%$ :

$$
\mathrm{SOC}=\frac{Q_{t}}{Q_{n}} \times 100 \%
$$

Here, $Q_{n}$ is the total battery power; $Q_{t}$ is the remaining battery capacity at the time of $t$, and formula can be expressed as following:

$$
Q_{t}=Q_{0}-\int_{0}^{t} i_{a}(t) \mathrm{d} t
$$

Here, $Q_{0}$ is the initial capacity of the battery, $\int_{0}^{t} i_{a}(t) \mathrm{d} t$ define the change of the battery capacity from the time of 0 to $t$.

Originally, some relatively simple algorithm was proposed, such as: integrate the current in amperes-time method [5], open circuit voltage method by means of OCV and SOC correspondence relationship and etc [6]. Then, the modified algorithm of these methods appeared, which added some or all amendments of the temperature, charge and discharge rate, charge and discharge efficiency. There are some advanced methods to be put for- 
ward as well, such as: fuzzy control algorithm, neural network algorithm [7], Kalman filter algorithm [8], and the newly emerged impedance spectroscopy method and C. Ehret's linear models method.

Although people pay a lot of effort to research this problem, the result is unsatisfactory. The mainly reason is that the battery's highly nonlinear. Various methods exist their shortcomings, such as: amperes-time method have error accumulation; open circuit voltage method is not suitable for current frequent fluctuation during driving; fuzzy control depend on engineering experience; neural networks rely on the choice of sample [9]; Kalman filtering depend on accurate battery and calculation complexity; impedance spectroscopy method requires additional function generator, increase costs; linear model method is only suitable for low current situation etc [10]. At present, generally combine the several algorithms to estimate SOC [11].

\section{ANFIS's Theorem and Structure}

\subsection{Combination the Fuzzy Technology and Neural Network}

Single neural network, just a black box system, is lacking of performance that could not provide the heuristic knowledge for the SOC's predication of battery. Therefore, It is not appropriate to express the knowledge based on the rules and not good to make use of the existing experience and knowledge. These drawbacks will increase the network's training time. A single fuzzy predictive can simply realize the learning heuristic knowledge but can't get the accurate result. Because the performance of self-learning and adaptive capacity is weak, it is difficult to form automatically and adjust the fuzzy rules of membership function local extremism. Combination of both can get the exact value at any conditions, and meanwhile can also understand the estimation process, can get the advantage of neural network and fuzzy systems.

In the fuzzy control system, fuzzy reasoning is a map to the relationship of input-output. Input as the premise (the error $\mathrm{E}$, the change rate of $\mathrm{E}$ and other fuzzy volume), makes the non-fuzziness control output. Since neurons can map any function relationships, it also can be used to make the fuzzy inference come true. In addition, the neural network can realize fuzziness and nonfuzziness. So that the neural network can represent all fuzzy control.we call this fuzzy control which is based on neural network. It has many advantages, such as computing and the number of knowledge experience are independent; allowed to contain a small amount of error experience (because it can be automatically excluded in the study)and can be parallel, distributed computing etc. Thus this fuzzy neural network, neural network-based fuzzy control, was used in hybrid electric vehicle energy management system [12].

\subsection{ANFIS's Construction}

Artificial Neuro-Fuzzy Inference Systems [13] is extensively used in the field of modeling, decision-making, signal processing and control. Here, the structure of ANFIS and its learning rule would be introduced.

Assuming that the fuzzy neural network has two inputs $x$ and $y$, one output $z$.

For the first-order Sugeno fuzzy model [14], the following rules:

Rule 1: If $x$ is $A_{1}$ and $y$ is $B_{1}$, then

$f_{1}=p_{1} x+q_{1} y+r_{1}$

Rule 2: If $x$ is $A_{2}$ and $y$ is $B_{2}$, then

$f_{2}=p_{2} x+q_{2} y+r_{2}$

The corresponding equivalent ANFIS model structure shown in Figure 1, the same floor node here has the same function.

Layer 1: $A_{1}$ and $B_{1}$ are input variables fuzzy sets, This layer node activation function on behalf of the membership function of fuzzy variables, the output represents fuzzy result called membership, one of the node transfer function can be expressed as:

$$
\begin{gathered}
O_{1, i}=f_{x i}(x) \quad i=1,2 \\
O_{1, j}=f_{y(j-2)}(y) \quad j=3,4
\end{gathered}
$$

Commonly the Gaussian function is used as the activation function.

Layer 2: multiply any two memberships which get by the fuzzy, so the output represents fuzzy rules or applicable degree of intensity.

$$
O_{2, i}=w_{i}=f_{x i}(x) f_{y i}(y) \quad i=1,2
$$

Layer 3: normalize each rule's apply degree:

$$
O_{3, i}=\bar{w}_{i}=\frac{w_{i}}{w_{1}+w_{2}} \quad i=1,2
$$

Layer 4: calculate each rule's conclusion:

$$
z_{i}=p_{i} x+q_{i} y+r_{i} \quad i=1,2
$$

Layer 5: Calculate the output of all rules and that the

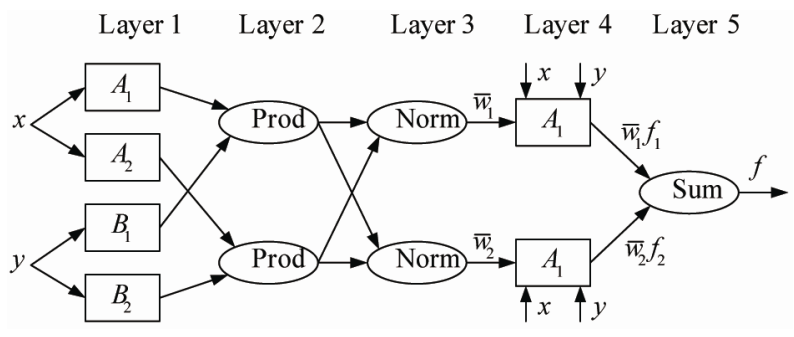

Figure 1. Equivalent ANFIS structure. 
output of the system output:

$$
z=\bar{w}_{1} Z_{1}+\bar{w}_{2} z_{2}
$$

$p_{i}, q_{i}, r_{i}$ are unknown, through the algorithm training, ANFIS can get them at a specified target to achieve the purpose of fuzzy modeling.

\section{SOC Estimation Based on ANFIS}

\subsection{SOC Estimation Model Based on ANFIS}

Hybrid operation process is very complicated. During the working process, the battery's SOC would be affected by many factors, such as: environmental temperature, initial voltage, battery resistance, working hours, and etc. The car will meet all kinds of problems in running, such as acceleration, climbing, cold, heat, rain, etc, as the battery power source is also influence by these conditions. Of course, the ideal neural network model is the more comprehensive input the better the output of the mapping, the more close to the actual conditions. However, many input data rely on a variety of instruments and sensors to get. More input data requires more costs, in light of this point and with the prerequisite of getting satisfied result, less input is good for result, which does not only reduce the difficulty of dealing with the problem, but also reduce costs.

In this paper, three inputs variables are available-the voltage $\mathrm{V}$, current $\mathrm{I}$, and cells surface temperature $T$, SOC's remaining battery charge percentage of capacity as only one output value is predicted, as shown in Figure 2. It is very difficult to realize the mapping from the three-dimensional space to one-dimensional by means of traditional method. For overcoming this problem, the appearance of development of fuzzy logic method is .to come with the tide of fashion. Through a large number of typical test data, the curve to extract some of the rules with regularity, that is human "work experience", and then use the fuzzy logic of the "reasoning" to achieve this "experience", it often can achieve better results. The design makes full use of fuzzy logic reasoning is simple, strong robustness and accuracy of neural network systems, and because neural network system for the three-

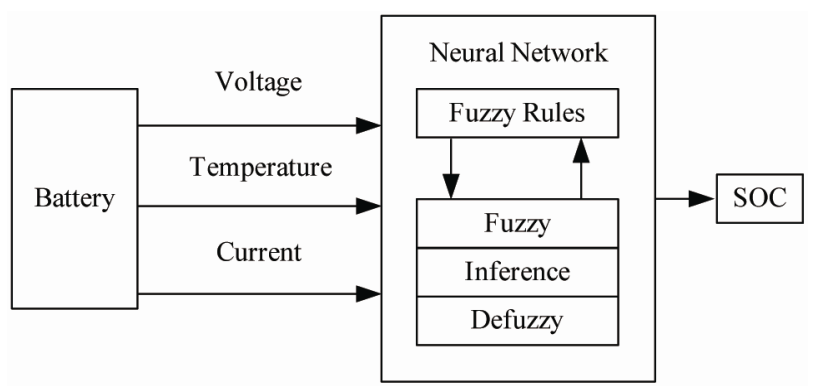

Figure 2. Adaptive fuzzy neural network prediction SOC values model diagram. input single-output system, making the hidden nodes is greatly reduced, easy to implement.

\subsection{SOC Estimates to Realize Based on ANFIS}

\subsubsection{Collect Data, Analyze and Create Data Sets and Test Data Sets}

At $22^{\circ} \mathrm{C}-27^{\circ} \mathrm{C}$ ambient temperature of the laboratory, we do constant flow duration discharge test for a manufacturer $3.3 \mathrm{~V}$ - $10.5 \mathrm{Ah} \mathrm{LiFePO}_{4}$ batteries SLFP-PT30, at the condition of $0.48 \mathrm{C}, 0.95 \mathrm{C}, 1.43 \mathrm{C}$ (i.e. $5 \mathrm{~A}, 10 \mathrm{~A}$, $15 \mathrm{~A}$ ). According to the relevant information of manufacturer to take $0.48 \mathrm{C}, 0.95 \mathrm{C}, 1.43 \mathrm{C}$ constant current battery discharge continued to discharge termination voltage is $3.18 \mathrm{~V}, 3.24 \mathrm{~V}, 3.28 \mathrm{~V}$.

The following is the fitting curve in the condition of different discharge current and battery voltage discharge time. It is shown in Figure 3.

It can be seen from the curves that the voltage's downward trend under the condition of large discharge current is faster than then the relatively small one.

\subsubsection{Determination the ANFIS Network Structure} In order to make use of MATLAB fuzzy toolbox anfis simulation of the data collected, we choose the function of genfisl inside. Function genfisl through the way of grid partition to given data set to generate a fuzzy inference system, which can be used in conjunction with the function anfis. By function genfisl generated the fuzzy inference system input and output membership function curves are to ensure that cover the entire input and output space evenly divided on the basis of its input and output membership functions of the type and number specified in the use, you can also use short provincial value.

Provide Training data and test data: With the different discharge rate of the voltage, current, SOC and time series. The odd items were regarded as the training data, and the even items as the authentication data.

\subsubsection{Determination the Type of Input and Output Membership Function}

Usually, ANFIS network could provide 8 variable pa-

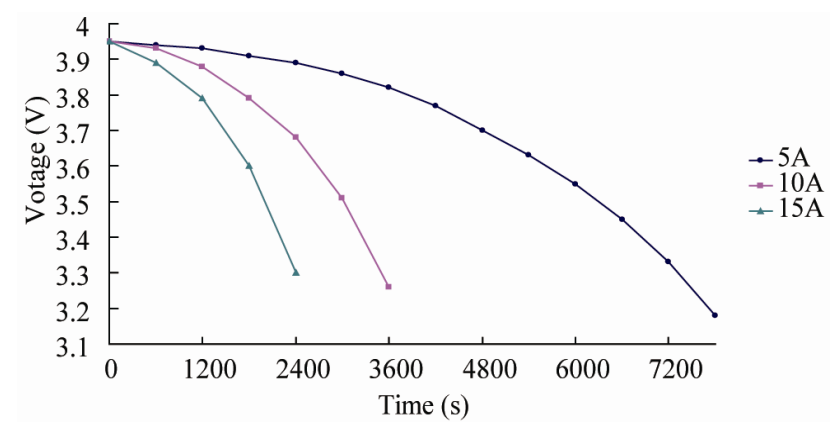

Figure 3. Different discharge current-voltage measurements. 
rameters of the function type MF. In the current paper, the Gaussian membership function (Gaussmf) is application [15]. Since ANFIS is a Sugeno type fuzzy system, so there are two output variables membership functions, namely: constant and linear functions. In this paper, constant, that the first order Sugeno fuzzy system.

\subsubsection{Divination Input Variable Space}

First determine the maximum and minimum input variables: then order the collected data to obtain the minimum and maximum input variables; finally, establish three fuzzy sets for each input variable, the corresponding generated results are high, low, medium membership functions, the input space is the input variables corresponding to the product of the membership. The output value from corresponding is between 0 and 1 .

\section{Experimental Validation and Analysis}

The selection of training data may give unreliable results bring some factors as before mentioned, This addition not only requires some pre-work on the availability of data, training process and the final result of model checking is also very important. In general, the result model test procedure is used for training those who do not use as the input/output data, to compare the model is or not trained to a very good match and predict these data.

The essence of BP algorithm is to solve the minimum problem of the error function, using steepest descent for nonlinear programming method, in accordance with the error function of the negative gradient direction to modify the weights, so it existence the disadvantage of low learning efficiency, slow convergence, and vulnerable Local minimum state, relatively poor network generalization ability.

The following is a BP network, ANFIS model comparison in predicted remaining battery capacity.

Figure 4 shows the compared curve between predictive value and measured one of the remaining capacity of 8 A constant discharge.

Figure 5 shows the remaining capacity under the 20A constant discharge predicted and measured values of the contrast curve.

According to the Figures $\mathbf{4}$ and $\mathbf{5}$, under the experimental condition, compared the SOC's predictive value and actual one, the majority of relative error could be controlled within $5 \%$. What's more, the predictive effect of ANFIS model is better and the error could be controlled within 3\%, which would not only meet the requirements of industrial applications, but is suitable to apply for the actual predictive research. And from the view of training steps and training time, ANFIS model to predict SOC is more efficient, ideal for real-time prediction.

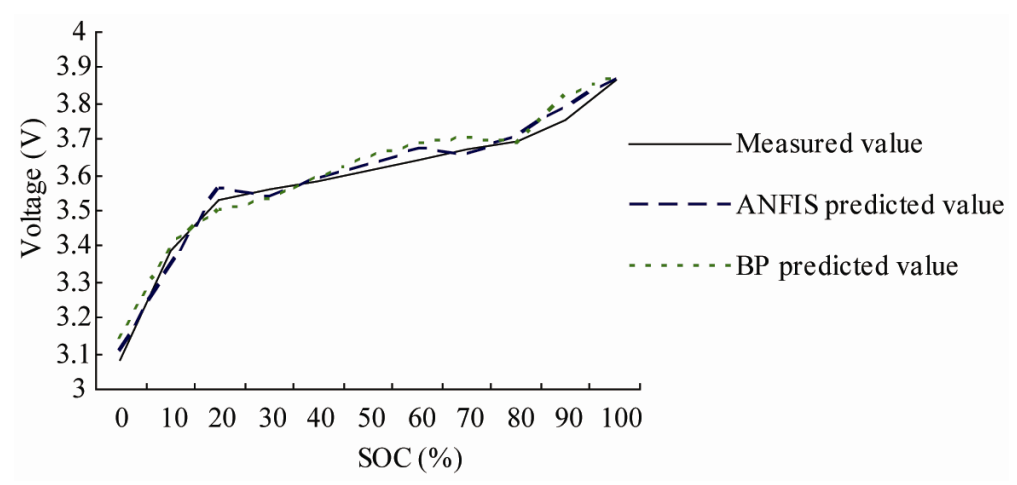

Figure 4. 8 A constant current discharge of the predictive value of SOC compared with the real.

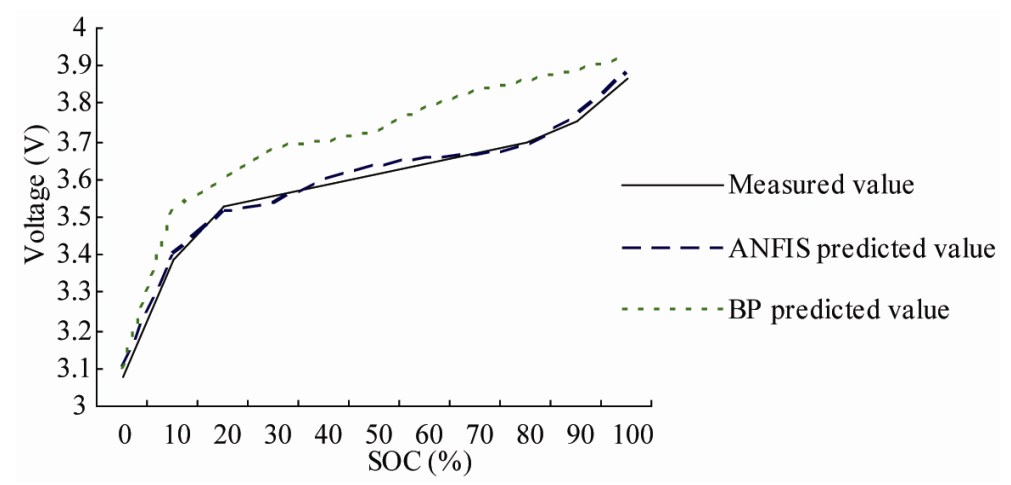

Figure 5. 20 A constant current discharge of the predictive value of SOC compared with the real. 


\section{Conclusion}

Based on the analysis of the traditional state of charge (SOC) estimation method, the current paper proposed a battery residual capacity model, featured with more generalization ability, adaptability and high precision. By analyzing the battery charge and discharge process, it is to determine the key parameters of SOC and then modify the experimental model in MATLAB platform. Through BP network prediction with comparison of experimental simulation of SOC values, indicating that the ANFIS has strong ability of adaptation and generalization, this method reduces the estimation error of SOC to less than 3\%, it can be used for intelligent monitoring system in hybrid car.

\section{REFERENCES}

[1] Z. Bin, "Voltage Characteristics of Li-Ion Power Battery for EVs,” Chinese Battery Industry, Vol. 14, No. 6, 2009, pp. 398-404.

[2] S. Pang, J. Farrell and J. Du, "Battery State-of-Charge Estimation," Proceedings of the American Control Conference, Arlington, 25-26 June 2001, pp. 1644-1649.

[3] J. Chiasson and B. Vairamohan, "Estimating the State of Charge of a Battery," American Control Conference, 4-6 June 2003, pp. 2863-2868. doi:10.1109/TCST.2004.839571

[4] B. Zhang, C. T. Lin and Q. S. Chen, "Performance of $\mathrm{LiFePO}_{4} / \mathrm{C}$ Li-Ion Battery for Electric Vehicle,” Chinese Journal of Power Sources, Vol. 32, No. 2, 2008, pp. 9598.

[5] L. C. Tao, W. J. Ping and C. Q. Shi, "Methods for State of Charge Estimation of EV Batteries and Their Application,” Battery Bimonthly, Vol. 34, No. 5, 2004, pp. 376378.

[6] S. J. Lee, J. H. Kim, J. M. Lee and B. H. Cho, "The State and Parameter Estimation of an Li-Ion Battery Using a New OCV-SOC Concept,” Power Electronics Specialists Conference, Orlando, 17-27 June 2007, pp. 2799-2803.

\section{doi:10.1109/PESC.2007.4342462}

[7] M. A. C. Valdez, J. A. O. Valera and M. J. O. Arteaga, "Estimating Soc in Lead-Acid Batteries Using Neural Networks in a Microcontroller-Based Charge-Controller," International Joint Conference on Neural Network, Vancouver, 30 October 2006, pp. 2713-2719.

[8] D. H. Feng, W. X. Zhe and S. Z. Chang, "State and Parameter Estimation of a HEV Li-ion Battery Pack Using Adaptive Kalman Filter with a New SOC-OCV Concept," International Conference on Measuring Technology and Mechatronics Automation, Zhangjiajie, 11-12 April 2009, pp. 375- 380.

[9] Q. Gang and C. Yong, "Neural Network Estimation of Battery Pack SOC for Electric Vehicles," Journal of Liaoning Technical University, Vol. 25, No. 2, 2006, pp. 230-233.

[10] A. R. P. Robat and F. R. Salmasi, "State of Charge Estimation for Batteries in HEV Using Locally Linear Model Tree (LOLIMOT)," Proceeding of International Conference on Electrical Machines and Systems, Seoul, 8-11 October 2007, pp. 2041-2045.

[11] T. X. Hui, D. H. Nan, F. Bo and Q. Y. Peng, "Research on Estimation of Lithium-Ion Battery SOC for Electric Vehicle," Chinese Journal of Power Sources, Vol. 134, No. 1, 2010, pp. 51-54.

[12] L. G. Hen, J. Hai and W. H. Ying, "The SOC Compute Model of Batteries Based on Fuzzy Neural Network," Journal of Test and Measurement Technology, Vol. 21, No. 5, 2007, pp. 405-409.

[13] Z. H. Li, H, L. Ping and Z. Z. Hua, "Study of Intelligent Prediction of the SOC of MH/Ni Battery for Electric Vehicle,” Machinery \& Electronics, No. 10, 2006, pp. 7-11.

[14] L. Y. Hong, "Sub Linearity of Generalized Sugeno Fuzzy Integrals,” Journal of Eastern Liaoning University (Natural Science), Vol. 17, No. 1, 2010, pp. 80-83.

[15] W. Tao, Q. Hao and C. Yang, “A New Method of Fuzzy Interpolative Reasoning Based on Gaussian-Type Membership Function,” Fourth International Conference on Innovative Computing Information and Control, Kaohsiung, 7-9 December 2009, pp. 966-969. 